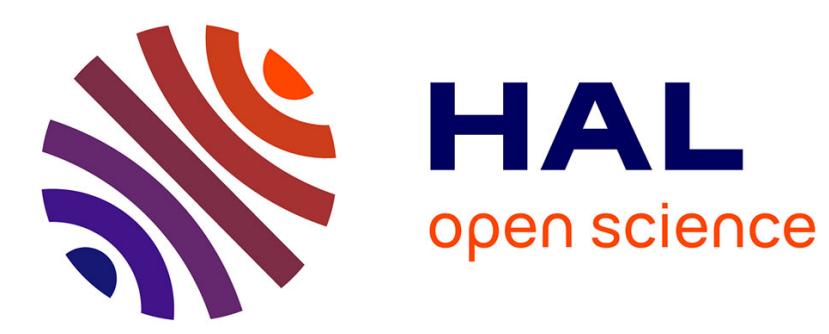

\title{
Early Detection of Atmospheric Turbulence for Civil Aircraft: A Data Driven Approach
}

Tianyi Li, Philippe Goupil, Josiane Mothe, Olivier Teste

\section{To cite this version:}

Tianyi Li, Philippe Goupil, Josiane Mothe, Olivier Teste. Early Detection of Atmospheric Turbulence for Civil Aircraft: A Data Driven Approach. 21st International Conference on Data Mining Workshops (ICDM 2021), Dec 2021, Auckland (virtual), New Zealand. pp.1087-1093, 10.1109/ICDMW53433.2021.00142 . hal-03556954

\section{HAL Id: hal-03556954 https://hal.science/hal-03556954}

Submitted on 4 Feb 2022

HAL is a multi-disciplinary open access archive for the deposit and dissemination of scientific research documents, whether they are published or not. The documents may come from teaching and research institutions in France or abroad, or from public or private research centers.
L'archive ouverte pluridisciplinaire HAL, est destinée au dépôt et à la diffusion de documents scientifiques de niveau recherche, publiés ou non, émanant des établissements d'enseignement et de recherche français ou étrangers, des laboratoires publics ou privés. 


\section{Early Detection of Atmospheric Turbulence for Civil Aircraft: A Data Driven Approach}

\author{
Tianyi Li \\ IRIT UMR5505 CNRS, Université de Toulouse \\ Aircraft Control, Airbus \\ Toulouse, France \\ Tianyi.Li@irit.fr
}

\author{
Philippe Goupil \\ Aircraft Control \\ Airbus \\ Toulouse, France \\ Philippe.Goupil@airbus.com
}

\author{
Josiane Mothe \\ IRIT UMR 5505 CNRS, INSPE \\ Université de Toulouse \\ Toulouse, France \\ Josiane.Mothe@irit.fr
}

\author{
Olivier Teste \\ IRIT UMR 5505 CNRS \\ Université de Toulouse \\ Toulouse, France \\ Olivier.Teste@irit.fr
}

\begin{abstract}
Atmospheric turbulence phenomena are the main causes of injuries in civil air transport and due to climate change, the frequency and severity of turbulence is increasing [1]. There is no precise turbulence prediction method [2]. The state-of-the-art turbulence detection methods used on board commercial aircraft include pilot reports and online algorithms based on in situ eddy dissipation rate. They provide turbulence observations but not their predictions. Weather radar on the other hand only detects turbulence in wet air without any precise announcement about the timing.

Equipped with a large number of sensors coming from different aircraft systems, the flight variables (multivariate time series generated by sensors) as well as their relationship may contain useful information indicating upcoming turbulence. Our approach aims at representing raw time series as functions which enable not only discovering the underlying function behind raw measurements but also implicitly removing data noise. Functional geometry features, which can capture the dynamic relation between variables, are deduced from the multidimensional path in functional representation. Based on the transformed geometry features, an outlier detection method is further deployed to detect specific behaviors indicating upcoming severe turbulence. Preliminary experimental results show that our approach reaches a 0.532 true positive rate while keeping a zero false positive rate, which meets the zero false alarm requirement for optimizing the passenger experience.
\end{abstract}

Index Terms-Atmospheric turbulence, Multivariate time series, Functional shape features, Early detection

\section{INTRODUCTION}

Atmospheric turbulence phenomena are the main causes of in-flight injuries in civil air transport. According to International Air Transport Association [1], turbulence is responsible for $25 \%$ of injuries in flight and causes $14 \%$ of flight diversions. In addition, the frequency and severity of turbulence is increasing due to the climate change [1]. Hence, there is a clear operational need to alert the crew before the aircraft crosses a turbulent zone. The goal is to alert the crew and the passengers sufficiently in advance to apply safety rules to

This work is sponsored by ANRT (CIFRE contrat $\mathrm{N}^{\circ} 2020-0205$ ). avoid injuries, as well as to quantify in advance the level of severity.

There is no precise and reliable prediction method for inflight turbulence [2]. The state-of-the-art turbulence detection methods used on board commercial aircraft include pilot reports which record turbulence events according to pilot subjective perception and in situ Eddy Dissipation Rate (EDR) algorithms which calculate the intensity of turbulence to alert the aircraft flying behind. Pilot reports and in situ EDR algorithms provide turbulence observations but do not predict it. Weather radar detects turbulence, but only in wet air and without any precise announcement about the timing. The existing detection techniques do not accurately quantify the level of turbulence nor predict it.

On board sensors coming from different aircraft systems (e.g. avionic systems, engines) generate multivariate time series, which allow data driven approaches to become possible options. Figure 1 shows the variation of on-board sensors' measurements while encountering severe turbulence. Flight variables exhibit strong fluctuation in the turbulence area. Atmospheric turbulence can be detected if these specific behaviors are early identified. In this work, the problem of turbulence detection is cast as a problem of rare event early classification task. We use time series data from sensors for this. Both the flight variables and their relationship change before encountering turbulence; this may provide valuable information indicating upcoming turbulence. To early detect atmospheric turbulence, we propose to utilize both flight variables and their relationship.

Most existing multivariate time series early classification methods do not consider the relationship among variables [3], [4]. He et al. [5] use a shapelet based early classification method that extracts core shapelets for each variable independently and relies on the combination of core shapelets to reflect the connection between variables. This method, however, does not consider the relationship among variables before shapelet extraction. To address this problem, we early classify upcom- 


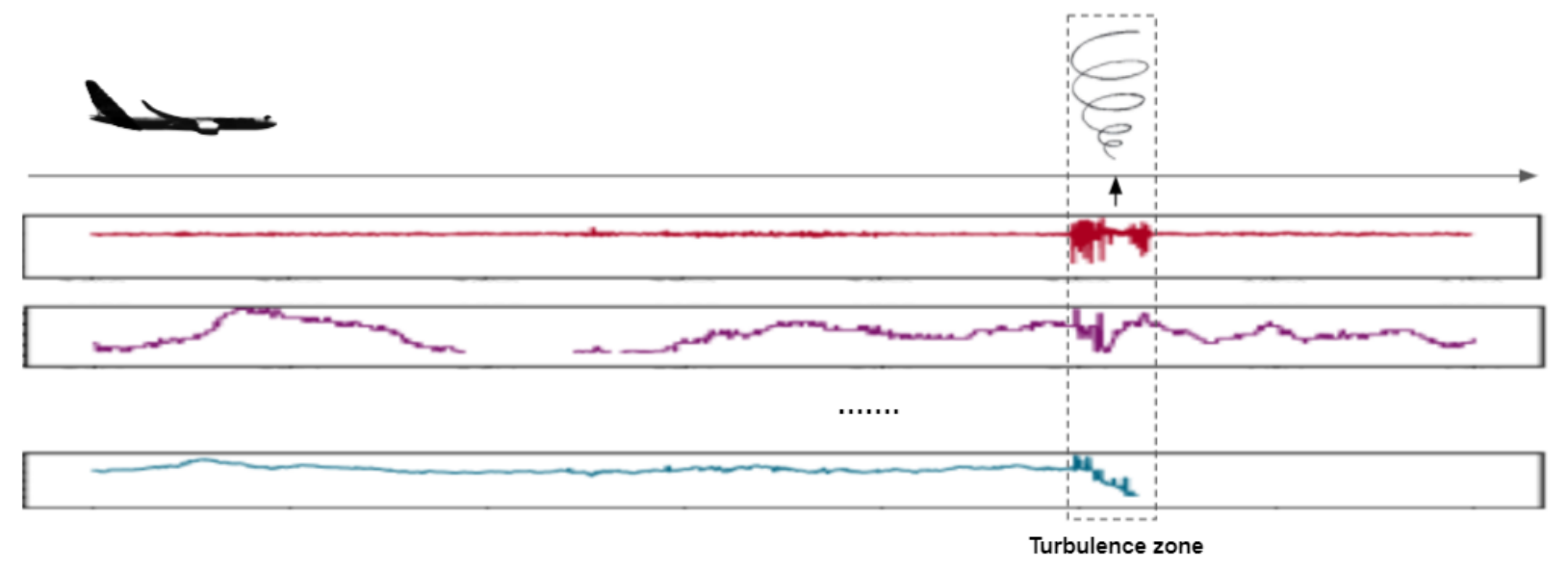

Variable 1

Variable 2

Variable $n$

Fig. 1. Variation of on-board sensors' measurements while encountering severe atmospheric turbulence. Flight variables exhibit specific behaviors in turbulence zone.

ing turbulence based on functional shape features extracted from the multidimensional path in functional representation, where each shape feature exhibits a different relationship between variables.

Our approach is based on Functional Data Analysis (FDA). As the time series coming from on board sensors are being recorded continuously, the data can be regarded as functional data. FDA encompasses the statistical methodology for such data (See [6] for general introductions to FDA). To detect upcoming turbulence information lying in the flight variables as well as their relationships, our approach represents raw time series in a functional form and extracts functional shape features based on the functional representation [7], [8]. Functional isolation forest [9] is further deployed based on the functional shape features to detect the upcoming turbulence. The advantages of the approach are as follows: 1. Functional representation enables discovering the underlying function behind noisy sensor data. 2 . Deduced functional shape features allow us to capture the dynamic correlation between variables. 3. By employing the functional shape features and considering turbulent signals as outliers compared with normal sensor signals, functional isolation forest can identify the specific behaviors indicating upcoming severe turbulence.

The paper is organized as follows: Section II introduces the problem and related works. Section III describes the proposed approach. The data preparation, experimentation settings, as well as the results achieved so far, are given in section IV. The conclusion and the plan for future works are discussed in section $\mathrm{V}$.

\section{Problem AND Related works}

Aircraft on board sensors generate multivariate time series. As sensors' data contains different levels of noise and turbulence events are rare compared with normal flights, early identification of atmospheric turbulence using sensor data can be specified as a "noised multivariate time series rare event early classification" problem. a) Multivariate time series early classification: Early classification of time series aims to predict the class label of incoming time series as early as possible [10]. It is applied in many domains such as earthquake warning [11] and gaz leakage detection [12]. To avoid serious consequences, atmospheric turbulence should be correctly identified with a certain amount of time in advance. As reviewed in [4], several shapelet based and prefix based multivariate early classification methods have been proposed in recent years.

Shapelet based methods search for time series subsequences that are representative of a class and appear early in time [5], [13]. Ghalwash et al. [14] developed a multivariate shapelets detection method for multivariate time series early classification. The proposed method extracted core shapelets that have the same start and end points for all variables. The authors in [15] proposed interpretable patterns for early diagnosis, which allowed multivariate shapelets with different start and end points. He et al. [5] obtained key shapelets for each variable separately and dealt with within-class imbalance problems by clustering shapelet candidates. The authors in [16] further proposed a method with interpretable rules which selected key shapelets by estimating the confidence of early classification for multivariate time series. To generate shapelets from categorical time series, Lin et al. [17] proposed an early classification approach for multivariate time series that contained categorical variables along with numerical variables. To address class imbalance problem in a multivariate time series early classification scenario, He et al. [18] combined an under-sampling method with shapelet based approaches and proposed an early prediction on imbalanced multivariate time series.

Prefix-based methods are dedicated to first learn minimum prediction length of time series and then utilize it for early classification [19]. Ma et al. [3] transformed multivariate time series into univariate time series by center sequence and executed the early classification on piecewise aggregate approximation representations. Gupta et al. [20] focused on multivariate time series from sensors with different sampling 
rates and proposed a divide-and-conquer based method.

Equipped with thousands of sensors on board commercial aircraft, the evolution of flight variables (see Figure 1) as well as their relationship may provide valuable information indicating upcoming turbulence. Most existing multivariate time series early classification methods do not consider these relationships [3], [4]. An early classification method proposed in [5] utilized the combination of core shapelets to reflect the connection between variables, but the relation among variables is not considered before shapelet extraction. To address this problem and capture relationships between the variables, we extract shape features form the functional representation of incomplete time series truncated several seconds or minutes before turbulence appears (See Section III).

b) Data Noise and different sampling frequency issues: Signals coming from on board sensors contain various levels of noise and variables also have different sampling frequencies, which will undoubtedly cause difficulties for the early classification task and thus demand time series smoothing or representation.

Conventional time series smoothing methods including exponential smoothing [21] and filter (butterworth filter, elliptic filter, low pass filter, etc) aim to reduce data noise, whereas time series representations intend to not only implicitly reduce noise but also reduce dimensions and provide new means for later prediction or classification tasks [22]. Discrete fourier transform [23], discrete wavelet transform [24], singular value decomposition [25] and piecewise aggregate approximation [26] are the classic representation methods. Based on piecewise aggregate approximation, adaptive piecewise constant approximation [27] allows segments to have different lengths. Symbolic aggregate approximation [28] represents time series by the string of successive symbols. Besides, FDA [6] considers the functions that generates raw signals and decomposes high dimensional measurements by basis functions (wavelet, b-spline, fourier...) in the predefined interval. Therefore, FDA representation can not only smooth raw signals but also handle the issue of different sampling frequencies among multiple variables [8].

To early detect atmospheric turbulence using variables' relationship, as well as remove data noise and handle different sampling frequency issues, a FDA-based outlier detection method [6]-[9] is adapted.

\section{FDA-BASED MULTIVARIATE TIME SERIES OUTLIER DETECTION FOR ATMOSPHERIC TURBULENCE EARLY IDENTIFICATION}

Functional Data Analysis (FDA) aims to represent high dimensional discrete observations as functions [6]. Observations from high rate sensors deployed in commercial aircraft are real continuous functions observed at discrete time points, thus can be treated as functional data. As sensor signals exhibit particular behaviors in the turbulence zone, turbulent signals are considered as outliers in magnitude and shape compared with normal sensor signals and multivariate functional outlier detection methods [7]-[9] are applied to identify turbulence several seconds or minutes in advance.

The overall workflow (See Figure 2) can be divided into four steps. For the sake of simplicity, a bivariate time series data set from [6] is employed to illustrate the framework.

1. Functional representation. In the first step, raw measurements are approximated by a linear combination of basis functions. We refer to [6] for the following inferences.

Suppose that we have a $M$-variable raw time series and each variable owns $n_{m}, m \in[1, M]$ sampling points. We approximate measurements $\left\{y_{m i}\right\}, i \in\left[1, n_{m}\right]$ through $K_{m}$ derivative basis functions $b_{m k}, k \in\left[1, K_{m}\right]$. The coefficients $c_{m k}, k \in\left[1, K_{m}\right]$ for basis functions $b_{m k}, k \in\left[1, K_{m}\right]$ can be computed by the following least square criteria:

$$
J\left(c_{m}\right)=\underset{c_{m}}{\operatorname{argmin}} \sum_{i=1}^{n_{m}}\left(y_{m i}-\sum_{k=1}^{K} c_{m k} b_{m k}\left(t_{m i}\right)\right)^{2}
$$

The estimate $\hat{c}$ can be computed as:

$$
\hat{c}_{m}=\underset{c_{m}}{\operatorname{argmin}}\left(\left\|y_{m}-B_{m} C_{m}\right\|^{2}\right)=\left(B_{m}^{T} B_{m}\right)^{-1} B_{m}^{T} y_{m}
$$

Where the matrix $B_{m}=\left\{b_{m k}\left(t_{m i}\right)\right\}_{i \in\left[1, n_{m}\right], k \in\left[1, K_{m}\right]}$ contains the values of all basis functions at measurement points. $C_{m}=\left\{c_{m k}\right\}, k \in\left[1, K_{m}\right]$ is the coefficient vector. To avoid over fitting, roughness penalty is added to balance two conflicting goals: bias and variance, at the same time.

$J\left(c_{m}\right)=\underset{c_{m}}{\operatorname{argmin}} \sum_{i=1}^{n_{m}}\left(y_{m i}-\sum_{k=1}^{K} c_{m k} b_{m k}\left(t_{m i}\right)\right)^{2}+\lambda_{m} \int\left[D^{q} \hat{f}_{m}(t)\right]^{2} d t$

where $\hat{f}_{m}(t)=\sum_{k=1}^{K} c_{m k} b_{m k}(t)$ is the approximation function and $D^{q}=\frac{d^{q}}{d t}$ is the $q^{t h}$ derivative. $\lambda_{m}$ is a hyperparameter that controls the weight of the penalty and can be computed by cross-validation. In this context, $\hat{c}$ is obtained by equating the first derivative of $J\left(c_{m}\right)$ to 0 with with respect to $C_{m}$ :

$$
\begin{aligned}
\hat{c}_{m} & =\underset{c_{m}}{\operatorname{argmin}}\left(\left(\left\|y_{m}-B_{m} C_{m}\right\|^{2}\right)+\lambda_{m} C_{m}^{T} R_{m} C_{m}\right) \\
& =\left(B_{m}^{T} B_{m}+\lambda_{m} R_{m}\right)^{-1} B_{m}^{T} y_{m}
\end{aligned}
$$

where $R_{m}=\int D^{q} b_{m j}(t) D^{q} b_{m l}(t) d t$ with $j, l \in\left[1, K_{m}\right]$.

2.Path lying in $M$-dimensional space. Once the raw curves are approximated by smooth functions, a space is formed by combining all the variables, where each variable represents an axis of the multidimensional space. Such representation is adopted in [8] to incorporate multivariate time series. In this space, the state evolving over time traces a path through the $M$-dimensional space thus each point represents a system's state. Figure 2 shows an example of a two-dimensional space plotted by combining two variables.

3. Functional shape features. As the path lying in the multidimensional space is the representation of all variables, 


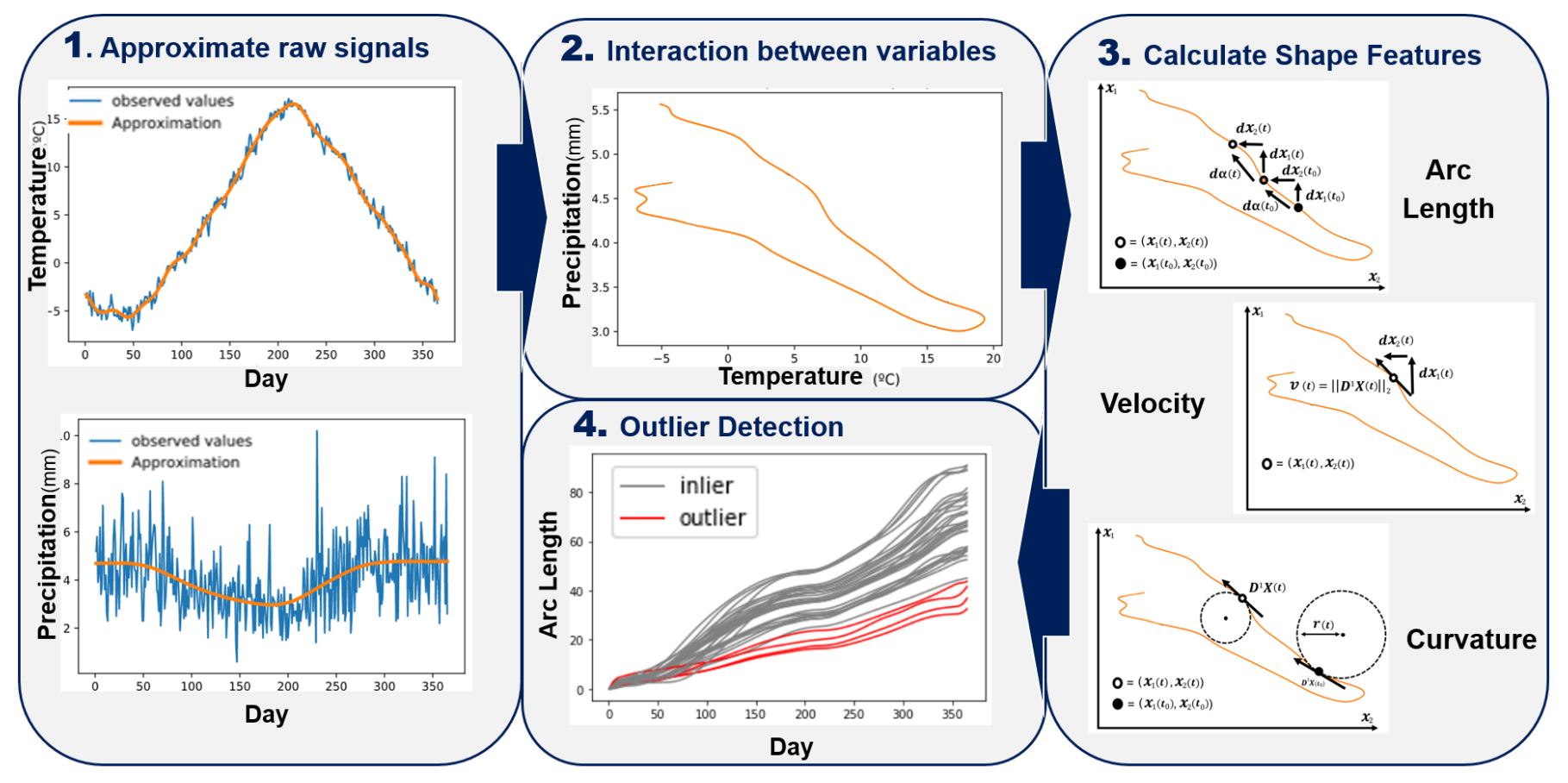

Fig. 2. Geometry based functional outlier detection method framework. The images for the first, second and fourth steps come from [29] and the illustrations for the third step are inspired by [8]. In the first step, raw signals (blue lines) are approximated by B-spline basis functions (orange lines). Then, in the second step, two variables are represented as a path in a two-dimensional space. In the third step, geometry shape features are extracted from the bi-dimensional path curve. This operation allows the shapes of the curves to capture the important relationships between variables to classify the time series. Then finally, in the fourth step, functional isolation forest is applied based on a shape feature to detect outliers.

its shape reflects the variables' relationship. Therefore, an underlying shape outlier in the path curve corresponds to a change of the relationship between variables. To capture shape features, various aggregation functions are applied, where each aggregation function extracts a shape feature and each shape feature exhibits a different aspect of relationship among variables. Aggregation functions are used in [7] to analyze multidimensional curves and the following shape features are adapted in this work as they have been successfully applied in [8] for multivariate time series outlier detection.

a) Arc length: Assume that $X(t)$ is a curve of a continuous variable $t$, the arc length $\alpha(t)$ is the cumulative length of $X(t)$ :

$$
\alpha(t)=\int_{t_{0}}^{t} \| D^{1}\left(X(u) \| d u=\int_{t_{0}}^{t} \sqrt{\sum_{k=1}^{M}\left(\frac{\mathrm{d} X(u)}{\mathrm{d} u}\right)^{2}} d u\right.
$$

where $\|$.$\| stands for the 12$-norm in $R^{M}$. Arc length is a positive increasing function which enable detecting the change of curve length. Thus, whether it is a continuous fluctuation or a sudden overshoot, as long as an outlier causes changes in the curve's length, it can be effectively revealed by arc length.

b) Velocity: Velocity shows the instantaneous change of the curve and can be regarded as the curve's slope at instant $t$

$$
\left.v(t)=\| D^{1} X(t)\right) \|
$$

Velocity is related to arc length with equations: $v(t)=\frac{\mathrm{d} \alpha}{\mathrm{d} t}$ or $\alpha(t)=\int_{t_{0}}^{t} v(t) d t$, which implies that velocity reflects the change of arc length.

c) Curvature: Curvature shows how quickly the tangent vector changes its direction. More intuitively, its inverse is the radius of the curve at instant $t$ and can detect outliers in bended shapes. Curvature is defined by the following equation:

$$
\kappa(t)=\frac{\left\|D^{1}\left(\frac{D^{1}(X(t))}{\| D^{1}(X(t) \|}\right)\right\|}{\| D^{1}(X(t) \|}
$$

where $\frac{D^{1}(X(t))}{\| D^{1}(X(t) \|}$ is the normalized tangent vector and $\left\|D^{1}\left(\frac{D^{1}(X(t))}{\| D^{1}(X(t) \|)}\right)\right\|$ can be regarded as its rate of change. Therefore, curvature is the rate of change of the tangent vector in relation to the normalized tangent vector.

Example. Figure 3 illustrates how a shape feature is used for turbulence early detection. It provides an example of arc length evolution before encountering atmospheric turbulence. A sliding window with a length of 1 minute and a step size of 10 seconds is performed. Red lines are flights that encounter turbulence while grey lines are the normal flights. One can see from the figure that the closer to the turbulence, the farther red lines are from grey lines. The increasing gap between red lines and grey lines not only indicates the incoming turbulence but also reflects the compromise between earliness and performance. In addition, it also shows that arc length is 


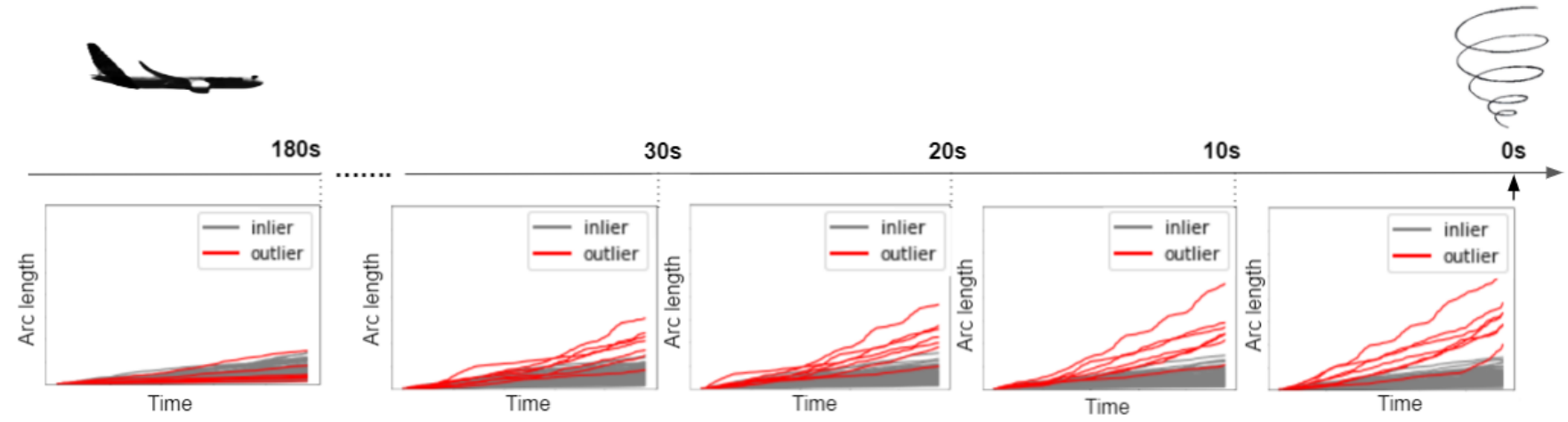

Fig. 3. Evolution of arc length before encountering atmospheric turbulence. Red lines are flights that encounter turbulence while grey lines are the normal flights. The closer to the turbulence, the farther red lines are from grey lines. The increasing gap between red lines and grey lines not only indicates the upcoming turbulence but also reflects the compromise between earliness and performance.

able to exhibit the early information of turbulence lying in the variables' relation.

Based on shape features, functional isolation forest is applied to further detect outliers.

4. Functional isolation forest. Functional isolation forest (FIF) [9] extends isolation forest (iForest) [30] from finite dimensional observations to functional data [9]. To split a node, each tree in iForest randomly selects a split variable among all variables and randomly selects a split value between the maximum and minimum values of the selected split variable [30]. In FIF, a dictionary $\mathcal{D}$ is predefined in a functional Hilbert space $\mathcal{H}$ equipped with a scalar product $\langle., .\rangle_{\mathcal{H}} \cdot \mathcal{D}$ can contain training data itself, stochastic elements (e.g. Brownian bridge dictionary) or deterministic functions (e.g. Cosine dictionary). Projection of a function $x \in \mathcal{H}$ on the dictionary $\mathcal{D},\langle x, d\rangle_{\mathcal{H}}(d \in \mathcal{D})$ defines a feature that describe $x$ [9]. Therefore, elements in dictionary $\mathcal{D}$ are the candidate split variables in FIF. Instead of randomly selecting a split variable, a probability distribution $\mathcal{V}$ on $\mathcal{D}$ is predefined to draw a split variable $d$. A split value is then uniformly drawn between the maximum and minimum values of the projections on $d$. Same as iForest, the path length from the root node to the terminal node reflects the normality of the instance in FIF, where anomalies would have shorter paths than normal samples.

Based on a shape feature, FIF returns an anomaly score for each instance; i.e., the bigger the score, the shorter the path, and the more likely the instance is an outlier (more likely that a flight will encounter severe turbulence in our case). How to set the threshold is tricky; i.e., a high threshold will cause more false negative cases whereas a low threshold will lead to more false alarms (false positive cases). For the purpose of having a robust turbulence alerting system, a relative high threshold is desired to eliminate false alarm. Moreover, to early identify severe turbulence, only time series before a certain timestamp are available. For example, if the earliness is set to 30 seconds, using arc length to predict turbulence means that only the diagrams before $30 \mathrm{~s}$ in Figure 3 are available. How to make the prediction as early as possible while ensuring the accuracy of the prediction will be further explored in future work.

\section{PRELIMINARY EXPERIMENTS}

\section{A. Data preparation}

The experiment data are collected from a general commercial aircraft model. All the flights have been labeled by domain experts into two classes: 'Turbulence' and 'Normal flights'.

As a preliminary experiment, we did not conduct a real time turbulence prediction. We use the sensors' recording extracted 30 seconds before severe turbulence happens and mix them with the normal flight signals. The goal of the model is to identify whether each flight will encounter turbulence in 30 seconds. Each flight contains the recording of 21 flight variables with same length (60s) but different sampling frequencies (from 8 hertz to 64 hertz). The data set contains 682 flights in total, among which 62 are the flights that encountered turbulence, and 620 are the normal flights.

\section{B. Experimental settings}

All variables are standardized to share the same scale. We use 300 B-spline functions of order five as basis functions for all variables considering that the data does not exhibit periodicity. The grid search of $\lambda_{m}$ is fixed on logarithmic scale in $[8,-6,-4,-2,0,2,4,6,8]$. Three different shape features are used in the experiment: arc length, velocity and curvature. For functional isolation forest, the number of functional isolation trees is set to 100 and Brownian bridge is used as dictionary. Sub-sampling size is set to 256.

Functional isolation forest is applied on three shape features respectively. To demonstrate the effectiveness of our approach, we also perform multivariate functional isolation forest directly on all 21 sensors variables.

\section{Preliminary results}

We use True Positive Rate (TPR) and False Positive Rate (FPR) to evaluate the detection and false alarm rates for turbulence alerting system. Here, detection rate means the percentage of real severe turbulence events that are successfully 
TABLE I

EXPERIMENT RESULTS

\begin{tabular}{|c|c|c|c|}
\hline & $\boldsymbol{T P R}$ & $\boldsymbol{F P R}$ & $\boldsymbol{A U C}$ \\
\hline Multivariate functional isolation forest - All 21 flight variables & 0.161 & 0.084 & 0.565 \\
\hline Functional isolation forest - Arc length & 0.694 & 0.031 & 0.943 \\
\hline Functional isolation forest - Velocity & $\mathbf{0 . 7 5 8}$ & $\mathbf{0 . 0 2 4}$ & $\mathbf{0 . 9 6 0}$ \\
\hline Functional isolation forest - Curvature & 0.629 & 0.037 & 0.896 \\
\hline
\end{tabular}

identified in advance and false alarm rate is the percentage of erroneous alerts among all the alerts. Besides, AUC (Area Under Curve) of ROC (Receiver Operating Characteristic) is also used for the evaluation as it shows models' general classification performance regardless of skew in class distribution and the change of threshold [31].

Table I shows the preliminary experimental results. In this experiment, the threshold of functional isolation forest is set to 0.091 .

Compared with applying multivariate functional isolation forest directly on all the flight variables, outlier detection based on shape feature (whether it is arc length, velocity or curvature) obtains much better result in both TPR, FPR and AUC, which demonstrates the effectiveness of our approach in early identifying in-flight turbulence. Velocity has the highest TPR and AUC as well as the lowest FPR, which may be due to its ability to capture the instantaneous changes in the relationship of variables that the upcoming turbulence information exhibits.

Considering our application scenarios and actual operational needs, a turbulence altering system should have a perfect operational robustness. This requires us to maximize TPR while keeping a zero FPR.

Figure 4 shows the ROC curve of turbulence early classification model using the shape feature velocity. Moving up from the bottom left corner of the curve, TPR can reach up to 0.532 with a zero FPR. This can be achieved by setting the threshold to 0.049 . From an operational point of view, such result is encouraging as there is currently no accurate prediction for in-flight turbulence. Nevertheless, how to develop a threshold setting strategy to better generalize the method requires more experimentation in future work.

\section{CONCLUSION AND FUTURE WORK}

The purpose of this research is to provide a new solution for atmospheric turbulence early detection. We use multivariate time series data coming from aircraft on board sensors and define a functional shape-based outlier early detection method. Our approach can not only capture the dynamic relation between variables, but also allows us to detect the upcoming turbulence information based on the shape of the transformed geometry features. The preliminary experiment was conducted on commercial aircraft flight recordings and the results showed that our approach achieved a true positive rate of 0.532 with zero false positives.

For future work, we will consider more shape features, as well as their combinations (e.g. by function composition).

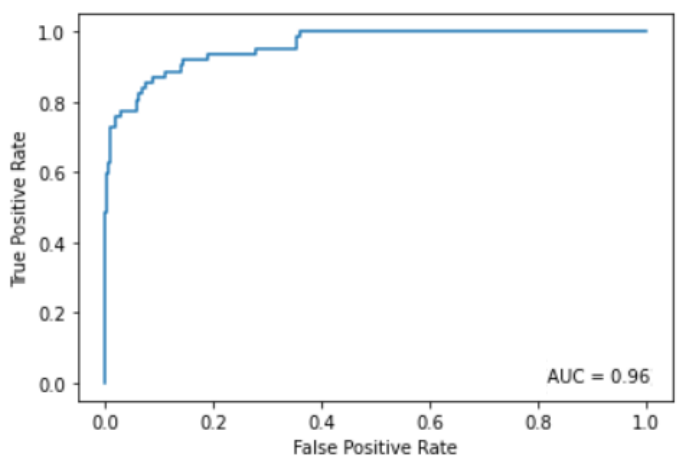

Fig. 4. ROC curve of the turbulence early classification model using the shape feature velocity

Furthermore, to generalize the model and ensure the same performance, a threshold setting strategy needs to be made. We plan to compare our approach with state-of-the-art multivariate time series early classification methods and we will also study how to apply the method in real time, as well as investigate the trade-off between earliness and performance.

\section{REFERENCES}

[1] IATA Turbulence Workshop, London, September 2018. International Air Transport Association, Montreal, Canada. www.iata.org.

[2] Sharman, R., Lane, T. (2016). Aviation Turbulence. Springer International Publishing, Switzerland, DOI, 10, 978-3.

[3] Ma, C., Weng, X., Shan, Z. (2017, October). Early classification of multivariate time series based on piecewise aggregate approximation In International Conference on Health Information Science (pp. 81-88). Springer, Cham.

[4] Gupta, A., Gupta, H. P., Biswas, B., Dutta, T. (2020). Approaches and applications of early classification of time series: A review. IEEE Transactions on Artificial Intelligence.

[5] He, G., Duan, Y., Peng, R., Jing, X., Qian, T., Wang, L. (2015). Early classification on multivariate time series. Neurocomputing, 149, 777787.

[6] Ramsay, J. O. (2004). Functional data analysis. Encyclopedia of Statistical Sciences, 4.

[7] Srivastava, A., Klassen, E. P. (2016). Functional and shape data analysis (Vol. 1). New York: Springer.

[8] Lejeune, C., Mothe, J., Soubki, A., Teste, O. (2020). Shape-based outlier detection in multivariate functional data. Knowledge-Based Systems, $198,105960$.

[9] Staerman, G., Mozharovskyi, P., Clémençon, S., d'Alché-Buc, F. (2019, October). Functional isolation forest. In Asian Conference on Machine Learning (pp. 332-347). PMLR.

[10] Mori, U., Mendiburu, A., Miranda, I. M., Lozano, J. A. (2019). Early classification of time series using multi-objective optimization techniques. Information Sciences, 492, 204-218.

[11] Fauvel, K., Balouek-Thomert, D., Melgar, D., Silva, P., Simonet, A., Antoniu, G., ... Termier, A. (2020, April). A distributed multi-sensor machine learning approach to earthquake early warning. In Proceedings 
of the AAAI Conference on Artificial Intelligence (Vol. 34, No. 01, pp. 403-411).

[12] Hatami, N., Chira, C. (2013, April). Classifiers with a reject option for early time-series classification. In 2013 IEEE Symposium on Computational Intelligence and Ensemble Learning (CIEL) (pp. 9-16). IEEE.

[13] Xing, Z., Pei, J., Yu, P. S., Wang, K. (2011, April). Extracting interpretable features for early classification on time series. In Proceedings of the 2011 SIAM international conference on data mining (pp. 247-258). Society for Industrial and Applied Mathematics.

[14] Ghalwash, M. F., Obradovic, Z. (2012). Early classification of multivariate temporal observations by extraction of interpretable shapelets. BMC bioinformatics, 13(1), 1-12.

[15] Ghalwash, M. F., Radosavljevic, V., Obradovic, Z. (2013, December) Extraction of interpretable multivariate patterns for early diagnostics. In 2013 IEEE 13th International Conference on Data Mining (pp. 201-210). IEEE.

[16] He, G., Zhao, W., Xia, X. (2019). Confidence-based early classification of multivariate time series with multiple interpretable rules. Pattern Analysis and Applications, 1-14.

[17] Lin, Y. F., Chen, H. H., Tseng, V. S., Pei, J. (2015, May). Reliable early classification on multivariate time series with numerical and categorical attributes. In Pacific-Asia Conference on Knowledge Discovery and Data Mining (pp. 199-211). Springer, Cham.

[18] He, G., Duan, Y., Qian, T., Chen, X. (2013, October). Early prediction on imbalanced multivariate time series. In Proceedings of the 22nd ACM international conference on Information Knowledge Management (pp. 1889-1892).

[19] Xing, Z., Pei, J., Philip, S. Y. (2009, June). Early prediction on time series: A nearest neighbor approach. In Twenty-First International Joint Conference on Artificial Intelligence.

[20] Gupta, A., Gupta, H. P., Biswas, B., Dutta, T. (2020). A divide-andconquer-based early classification approach for multivariate time series with different sampling rate components in iot. ACM Transactions on Internet of Things, 1(2), 1-21.

[21] Brown, R. G., Meyer, R. F. (1961). The fundamental theorem of exponential smoothing. Operations Research, 9(5), 673-685.

[22] Wang, X., Mueen, A., Ding, H., Trajcevski, G., Scheuermann, P., Keogh, E. (2013). Experimental comparison of representation methods and distance measures for time series data. Data Mining and Knowledge Discovery, 26(2), 275-309.

[23] Agrawal, R., Faloutsos, C., Swami, A. (1993, October). Efficient similarity search in sequence databases. In International conference on foundations of data organization and algorithms (pp. 69-84). Springer, Berlin, Heidelberg.

[24] Chan, K. P., Fu, A. W. C. (1999, March). Efficient time series matching by wavelets. In Proceedings 15th International Conference on Data Engineering (Cat. No. 99CB36337) (pp. 126-133). IEEE.

[25] Faloutsos, C., Ranganathan, M., Manolopoulos, Y. (1994). Fast subsequence matching in time-series databases. ACM Sigmod Record, 23(2), 419-429.

[26] Keogh, E., Chakrabarti, K., Pazzani, M., Mehrotra, S. (2001). Dimensionality reduction for fast similarity search in large time series databases. Knowledge and information Systems, 3(3), 263-286.

[27] Keogh, E., Chakrabarti, K., Pazzani, M., Mehrotra, S. (2001, May). Locally adaptive dimensionality reduction for indexing large time series databases. In Proceedings of the 2001 ACM SIGMOD international conference on Management of data (pp. 151-162).

[28] Lin, J., Keogh, E., Wei, L., Lonardi, S. (2007). Experiencing SAX: a novel symbolic representation of time series. Data Mining and knowledge discovery, 15(2), 107-144.

[29] Guillaume Bernard, Clément Lejeune, Sandra Ferrieres and Olivier Teste, (2020), curve_shape_analysis, GitHub repository, https://github.com/Clej/curve_shape_analysis.git

[30] Liu, F. T., Ting, K. M., Zhou, Z. H. (2008, December). Isolation forest In 2008 eighth ieee international conference on data mining (pp. 413422). IEEE.

[31] Bradley, A. P. (1997). The use of the area under the ROC curve in the evaluation of machine learning algorithms. Pattern recognition, 30(7), 1145-1159. 